\title{
Proapoptosis Effect of Root of Eurycoma longifolia (Pasak Bumi) on the Prostate Cancer: In Silico Analysis
}

\author{
Eka Yudha Rahman*1,2, Mulyohadi $\mathrm{Ali}^{3}$, Basuki Bambang Purnomo ${ }^{4}$, Nia Kania ${ }^{5}$ \\ ${ }^{1}$ Doctoral Program of Medical Science, Faculty of Medicine, University of Brawijaya Malang, Indonesia \\ ${ }^{2}$ Department of Surgery, Urology Division, Faculty of Medicine, University of Lambung Mangkurat Banjarmasin, Indonesia \\ ${ }^{3}$ Department of Pharmacology, Faculty of Medicine, University of Brawijaya Malang, Indonesia \\ ${ }^{4}$ Department of Urology, Faculty of Medicine, University of Brawijay a Malang, Indonesia \\ ${ }^{5}$ Department of Anatomical Pathology, Urology Division, Faculty of Medicine, University of Lambung Mangkurat Banjarmasin, \\ Indonesia
}

\begin{abstract}
This study aimed to predict the proapoptosis effect of E. longifolia active compounds on prostate cancer by in silico analysis. Protein data such as BCL-2 (GI: 2506216), Caspase 3 (GI:_6978605), Caspase 8(GI: 11560103), data quassinoid (ID: 5459060 and chantin (ID: 97176) were collected from GenBank of NCBI. Protein BCL-2 collected from NCBI compare with Protein Data Bank (PDB) and UNIPROT. The docking process was carried out using software HEX 8.0. to compute the binding affinity between ligands (active compounds of Pasak Bumi) and protein target. The interaction between quassinoid and chantin was strongest and stable against caspase-9, indicating that the active ingredient in E. longifolia triggered caspase-9 activity after activation of $\mathrm{BH} 3$ domains in $\mathrm{Bcl}-2$ in prostate cancer. The low energy binding between quassinoid and chantin with caspase-3 indicates the interaction between the active ingredients is not strong with caspase-3. E. longifolia active ingredients that are potentially used in the treatment of prostate cancer are quassinoid and chantin by inducing apoptotic mechanisms via both extrinsic and intrinsic pathways. The combination of active ingredients of E. longifolia that is quassinoid and chantin can be used as a strategy of prostate cancer therapy both through extrinsic and intrinsic pathways.
\end{abstract}

\section{Introduction}

Prostate cancer will occur when immortal cells develop in the prostate gland. It was frequently diagnosed in men and become the fourth most common cancer in the world within 2014. In Indonesia, it has a high prevalence of occurrence which is about 16 cases in 100.000 men. Furthermore, Indonesia has an endemic plant called pasak bumi (Eurycoma longifolia Jack) which is well-known as traditional medicine and cancer therapy. The root extract of Pasak Bumi consists of eurycomanone, quassinoid, and canthin. Therefore, this study aimed to predict the proapoptosis effect of $E$. longifolia active compounds on prostate cancer by in silico analysis.

\section{Research Methods}

Protein data such as BCL-2 (GI: 2506216), Caspase 3 (GI:_6978605), Caspase 8(GI: 11560103), and data quassinoid (ID: 5459060 dan chantin (ID: 97176 ) were collected from GenBank National Center for Biotechnology Information (NCBI). Protein BCL-2 collected from NCBI compare with Protein Data Bank (PDB) and UNIPROT. The docking process was carried out using software HEX 8.0. to compute the binding affinity between ligands (active compounds of Pasak Bumi) and protein target. Each active compound was docked to the specific active site of a protein target. All biomolecules were visualized using software Discovery Studio 4.0. to generate the representative figure.

\section{Results and Discussion}

The interaction between quassinoid and chantin was strongest and stable against caspase-9, indicating that the active ingredient in E. longifolia triggered caspase-9 activity after activation of $\mathrm{BH} 3$ domains in $\mathrm{Bcl}-2$ in prostate cancer. Previous studies have suggested that $\mathrm{BH} 3$ peptides interact with $\mathrm{Bcl}-2$ in prostate cancerinducing apoptosis involving caspase-9 activation and followed by caspase-3 activation [1]. The phase of apoptotic execution involves the activation of several series caspases. Upstream caspase of the intrinsic pathway is caspase-9 while the extrinsic pathway is caspase 8. The intrinsic and extrinsic pathways will converge to activate caspase- 3 which plays a role in the apoptotic nuclear effector [2]. Direct activation of the execution caspase can be used in anticancer therapeutic strategies by increasing the concentration of procaspase-3 [3].

The low energy binding between quassinoid and chantin with caspase-3 indicates the interaction between the active ingredients is not strong with caspase-3. It is predicted that the active ingredient of E. longifolia does not directly interact with caspase- 3 in the apoptotic process of prostate cancer but caspase- 3 activation is affected by caspase- 9 .

Corresponding author: eyurologi05@gmail.com 

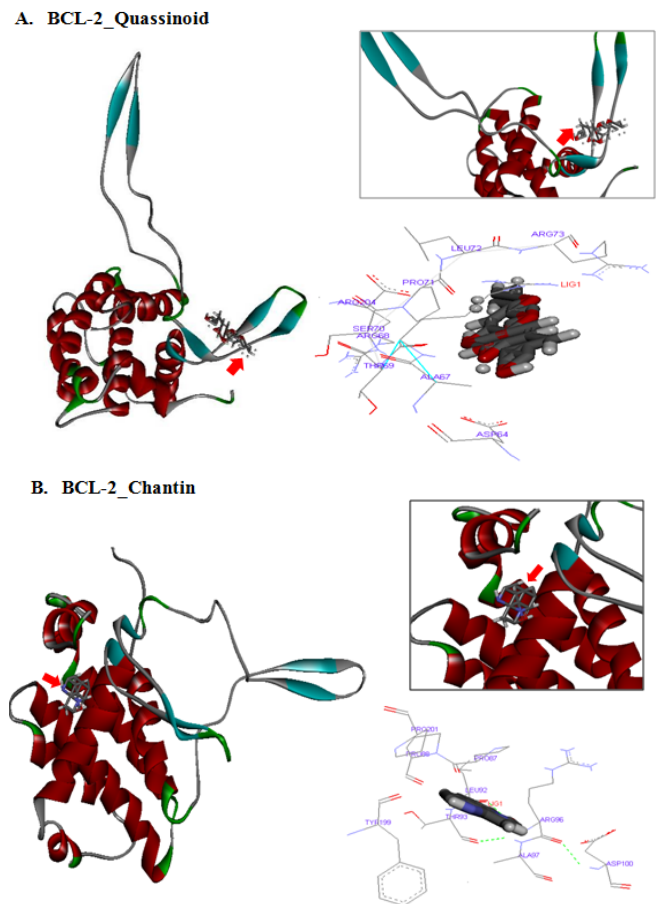

Fig.1 Interaction BCL-2 with Eurycoma longifolia compound. A. Quassinoid, B. Chantin FADD_Quassinoid
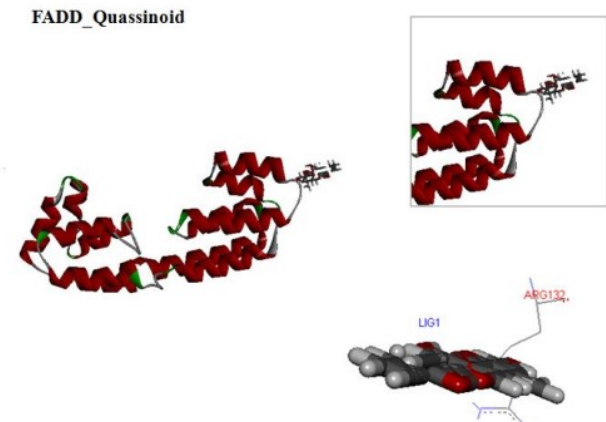

Fig. 2 Interaction between quassinoid and FADD

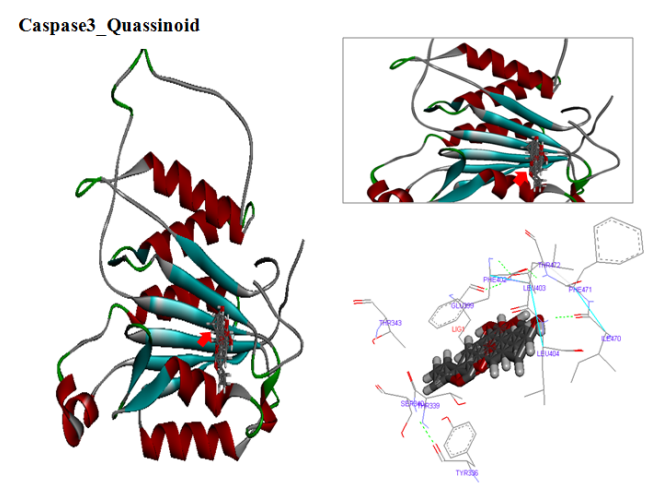

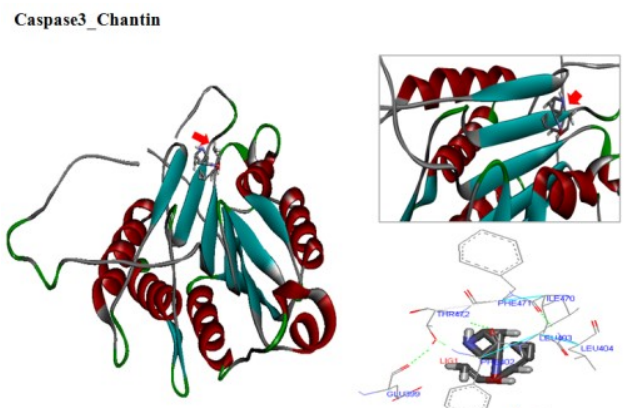
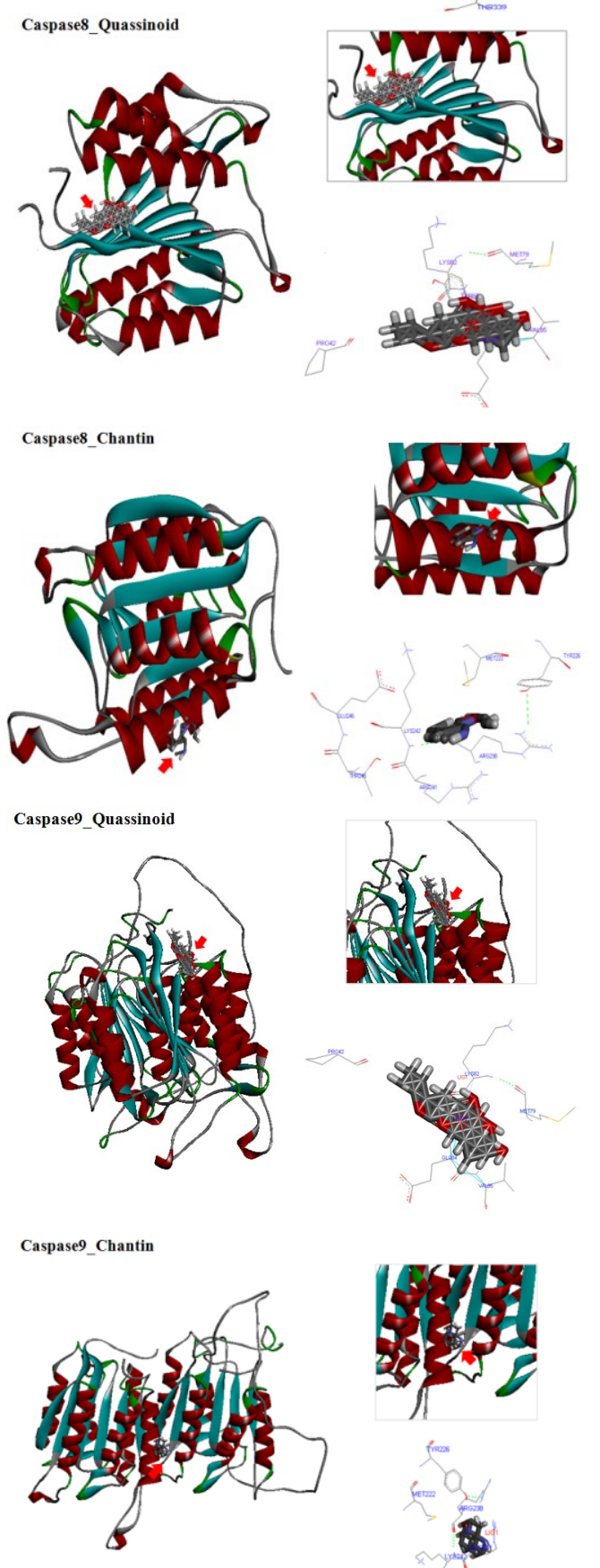

Fig. 3 Interaction quassinoid and chantin with caspase 3, caspase 8 , and caspase 9 . 


\section{Conclusion}

E. longifolia active ingredients that are potentially used in the treatment of prostate cancer are quassinoid and chantin by inducing apoptotic mechanisms via both extrinsic and intrinsic pathways. Chantin interacts directly with $\mathrm{BH} 3 \mathrm{Bcl}-2$ domains that act as proapoptosis, but with the stability of ligand and receptor interactions, quassinoid has a more stable bond but does not interact directly with $\mathrm{BH} 3$ domains. The activation of the BH3 pro-apoptotic domain in Bcl-2 affects the activation of cascade caspase. Quassinoid has interaction with FADD receptors and then activates caspase-8 (extrinsic pathway). Quassinoid and chantin have a strong interaction with caspase-9 then activate caspase-3 (intrinsic pathway). It was concluded that the combination of active ingredients of $\mathrm{E}$. longifolia that is quassinoid and chantin can be used as a strategy of prostate cancer therapy both through extrinsic and intrinsic pathways.

\section{References}

1. J.M. Jurgensmeier, Z. Xie, Q. Deveraux , L. Ellerby, D. Bredesen, J.C. Reed, Proc. Natl. Acad. Sci. USA, 95, 4997-5002, (1998)

2. M.C. Zhang, H.P. Liu, L.L. Demchik, Y.F. Zhai, D.J. Yang, Cell Res., 14 : 117-124, (2004)

3. K.S. Putt, G.W. Chen, J.M. Pearson, J.S. Sandhorst, M.S. Hoagland, J.T. Kwon, Nat. Chem. Biol., 2: 543-550, (2006) 